\title{
Aspergilosis invasora pulmonar en paciente con Leucemia Mieloide aguda en quimioterapia
}

\author{
Invasive pulmonary aspergillosis in patients with leukemia \\ Acute Myeloid chemotherapy \\ Verónica Contreras ${ }^{1 *}$, Mariajosé García ${ }^{1}$, Bernardita Rojas $^{2}$ \\ ${ }^{1}$ Becada Medicina Interna Hospital Carlos van Buren, Valparaíso. \\ ${ }^{2}$ Hematología Hospital Carlos van Buren. \\ *Autor para correspondencia: veronicacontreras.29@me.com
}

RECIBIDO:30 de Mayo de 2016

APROBADO:21 de Junio de 2016

LAS AUTORAS DECLARAN NO TENER CONFLICTO DE INTERESES

Palabras clave: aspergillosis, neutropenia, galactomanano.

Key word: aspergillosis, neutropenia, galactomannan.

\section{RESUMEN}

Se reporta un caso clínico de un paciente masculino de 65 años, que ingresó al hospital por cuadro de 10 días de evolución, con sospecha clínica y de laboratorio de un síndrome mieloproliferativo. Estudiado por hematología, se confirmó Leucemia Mieloide Aguda M5. Se inició quimioterapia de inducción. El paciente evolucionó con pancitopenia, destacando neutropenia severa hasta $200 / \mathrm{mm} 3$, febril y sin foco precisado. Se trató con antibióticos de amplio espectro por 10 días con buena respuesta. Cinco días después de finalizar su tratamiento antibiótico, nuevamente comenzó con fiebre, alza de parámetros inflamatorios, neutropenia severa y clínica de foco respiratorio. Se realizó Tomografía Computada (TC) de tórax y galactomanano en sangre, ambos compatibles con aspergilosis pulmonar, por lo que se inició tratamiento con voriconazol. El paciente evolucionó con buena respuesta clínica y de laboratorio, mejoría de imágenes del TC de tórax y negativización de galactomanano. Al mes cedió la pancitopenia. Fue dado de alta en buenas condiciones generales, con indicación de volver a hospitalizar para quimioterapia de consolidación.

\section{ABSTRACT}

A report of a clinical case of a male patient aged 65 is presented. He entered to the hospital for 10 days evolution box with clinical and laboratory suspicion of a myeloproliferative syndrome. Studied by hematology, acute myeloid leukemia M5 was confirmed. induction chemotherapy began. The patient developed pancytopenia, highlighting severe neutropenia up to $200 / \mathrm{mm} 3$, fever and without pointed focus. He was treated with broadspectrum antibiotics for 10 days with good response. Five days after finishing his antibiotic treatment began with fever again, rising inflammatory parameters, neutropenia and severe respiratory clinical focus. Computed Tomography (CT) of the chest and blood galactomannan was realized, both were compatible with pulmonary aspergillosis, starting treatment with voriconazole. The patient developed 
good both clinical and laboratory, improvement in chest $\mathrm{CT}$ images and negativization galactomannan response. Month later yielded pancytopenia. He was discharged in good general condition, indicating again been hospitalized for consolidation chemotherapy.

\section{INTRODUCCIÓN}

Los hongos pertenecientes al genero Aspergillus se encuentra ampliamente distribuidos por todo el mundo, son ubícuos en la naturaleza y la inhalación de conidias es un fenómeno habitual ${ }^{1}$. Entre sus numerosas especies, Aspergillus fumigatus es el causante de aproximadamente el $80 \%$ de las infecciones en humanos ${ }^{2}$.

Desde un punto de vista clínico, el término aspergilosis incluye enfermedades de diferente patogenia. El pulmón es el principal órgano afectado debido a la alta capacidad esporulativa de estos hongos y a que sus conidias son lo suficientemente pequeñas para poder alcanzar los alveolos ${ }^{2}$. La enfermedad pulmonar tiene un espectro de síndromes clínicos, como son el aspergilloma, la aspergilosis broncopulmonar alérgica, las formas pulmonares crónicas o semi invasoras, la aspergilosis pulmonar invasora (API) y las formas extrapulmonares y/o diseminadas ${ }^{1,3}$.

Las diferentes formas clínicas derivadas de la presencia de Aspergillus spp en la vía aérea deben verse como un proceso evolutivo, que se inicia con la colonización de la vía aérea, continúa con la ruptura de la barrera epitelial por las hifas del hongo (evento inicial de la invasión tisular), progresando a la invasión del parénquima pulmonar, pudiendo posteriormente diseminarse al resto del organismo ${ }^{4}$.

Sin embargo, la invasión tisular es infrecuente y ocurre fundamentalmente en pacientes con enfermedades hematológicas, neutropénicos o sometidos a algún grado de inmunosupresión ce- $\operatorname{lular}^{1,5}$. La incidencia es de hasta un $24 \%$ para los pacientes con leucemia aguda ${ }^{6,7}$, hasta un $10 \%$ en pacientes con trasplante de células madre hematopoyéticas alogénicas, y hasta un $7 \%$ en pacientes con malignidades linfoides ${ }^{8}$.

La API es una causa importante de morbimortalidad en este tipo de paciente. La tasa de mortalidad de API supera el 50\% en pacientes neutropénicos y alcanza el $90 \%$ en trasplante hematopoyético de células madre dependiendo del momento del diagnóstico y del tratamiento oportuno ${ }^{9}$. Se consideran pacientes de alto riesgo de desarrollo de API aquellos con una neutropenia severa mayor de 14 días, pacientes con leucemia mieloide aguda o síndrome mielodisplásico en tratamiento de inducción o en tratamiento de rescate por recaída o refractariedad, los receptores de trasplantes de células madre hematopoyéticas alogénicas o con enfermedad injerto contra huésped grave asociada y aquellos con el uso prolongado de corticosteroides ( $>2$ semanas) y / o en dosis altas ( $\geq 2 \mathrm{mg} / \mathrm{kg}$ ) o inmunodepresores de células $T^{8,10}$.

El diagnóstico de la API sigue siendo un reto. El diagnóstico precoz en pacientes severamente inmunocomprometidos es difícil, y es necesario un alto índice de sospecha en pacientes con factores de riesgo para la enfermedad invasora ${ }^{9}$.

El diagnóstico de API en etapas iniciales resultaría en el comienzo de terapia antifúngica apropiada cuando la carga fúngica es aún baja, lo que podría mejorar el pronóstico ${ }^{11}$.

\section{CASO CLÍNICO}

Paciente masculino de 65 años de edad, residente de Valparaíso (Chile), con antecedentes de cáncer de próstata diagnosticado y tratado con quimioterapia y radioterapia en el año 2014. Ingresa a Unidad de Emergencias de Hospital Carlos van Buren el día 19 de marzo del 2016, por cuadro clínico de 10 días de evolución, caracterizado por 
compromiso del estado general, dolor abdominal, sensación febril, lesiones equimóticas en piel y gingivorragía. En los exámenes de laboratorio de ingreso destacó leucocitosis de $30.000 / \mathrm{mm}^{3}$, con desviación izquierda hasta promielocitos, $12 \%$ de blastos y eritroblastos, trombocitopenia e hiperbilirrubinemia de predominio indirecto. Se realizó TC de tórax y abdomen que mostró nódulos pulmonares inespecíficos, nefrolitiasis izquierda no obstructiva, hernia inguinal izquierda sin signos de complicación, hígado y bazo normales con vía biliar fina. Se hospitalizó para completar estudio.

Se realizó un hemograma de control que mostró hemoglobina de $10 \mathrm{gr} / \mathrm{dl}$, leucocitosis $14.000 / \mathrm{mm}^{3}$, con desviación izquierda hasta mielocitos, $44 \%$ de blastos grandes microgranulares, $12 \%$ eritroblastos y trombocitopenia de 36.000/ $\mathrm{mm}^{3}$, lactato deshidrogenasa $2587 \mathrm{U} / \mathrm{L}$ y proteína $\mathrm{C}$ reactiva elevada (PCR).

Fue evaluado por equipo de Hematología para estudio de síndrome mieloproliferativo. Se tomó mielograma que resultó sin fragmentos, pero dado hemograma y frotis sanguíneo sugerente de Leucemia Mieloide Aguda, se envió muestra de sangre periférica para estudio de inmunofenotipo, citogenética y biologia molecular.

Paciente se trasladó a Unidad de Cuidados Intermedios para continuar estudio y manejo el día 22/03/16. Se inició quimioterapia de inducción (Daunorrubicina/Citarabina) el día 24/03/16. El informe de inmunofenotipo confirmó Leucemia Mieloide Aguda M5. El día 25/03/16 presentó fiebre, alza de parámetros inflamatorios (PCR 135 $\mathrm{mg} / \mathrm{L}$ ) y leucopenia $1.200 / \mathrm{mm}^{3}$. Se trató durante 10 días con Tazonam sin lograr precisar foco. Dos hemocultivos negativos. Paciente presentó buena evolución clínica y de parámetros de laboratorio. Se mantuvo con pancitopenia durante casi un mes (desde ingreso), alcanzando leucopenia hasta 200/ $\mathrm{mm}^{3}$, y siendo transfundido con glóbulos rojos y plaquetas en varias ocasiones.
El día 08/04/16 nuevamente cursó con fiebre y alza de los parámetros de actividad inflamatoria (PCR $319 \mathrm{mg} / \mathrm{L}$ ), glóbulos blancos: 300/ $\mathrm{mm}^{3}$, con TC de tórax con lesión condensante lóbulo pulmonar superior izquierdo con signo del halo (Imagen 1) y GM positivo (índice 0.52). Paciente evolucionó hipotenso, con falla respiratoria moderada, por lo que requirió oxigenoterapia y kinesioterapia respiratoria.

Se inició precozmente tratamiento con imipenem/vancomicina por 10 días y voriconazol durante 22 días. TC de control a los 4 días (imagen 2) y a los 10 días (Imagen 3) de tratamiento antibiótico-antimicótico, con aumento del tamaño de la lesión pulmonar, derrame pleural bilateral mayor a izquierda. Posterior a esto, paciente evolucionó con muy buena respuesta clínica y de laboratorio, los índices de actividad inflamatoria bajaron y la falla respiratoria se recuperó. Hemocultivos (cuatro) negativos. Hubo negativización de GM (índice 0.08 el 19/04/16). El día 18/04/16 se recuperó de su pancitopenia, con recuento absoluto de neutrófilos de $2.772 / \mathrm{mm}^{3}$, hemoglobina $9.4 \mathrm{gr} / \mathrm{dl}$, plaquetas $148.000 / \mathrm{mm}^{3}$. Durante el periodo de tratamiento con voriconazol hizo una reacción adversa a medicamento, presentó elevación significativa de transaminasas y coagulopatía, que mejoró con administración de 3 dosis de Vitamina K endovenosa.

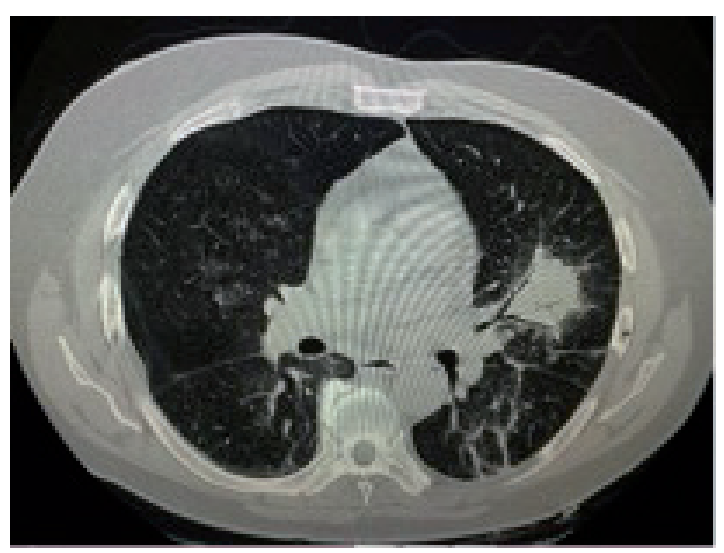

Figura 1. TC de tórax con lesión condensante lóbulo pulmonar superior izquierdo con signo del halo. 


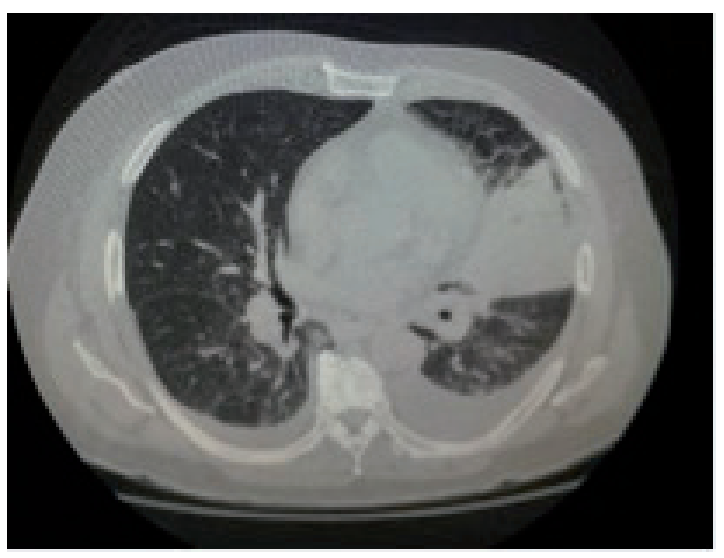

Figura 2. TC de control a los 4 días, se observa aumento de la lesión condensante con derrame pleural bilateral.

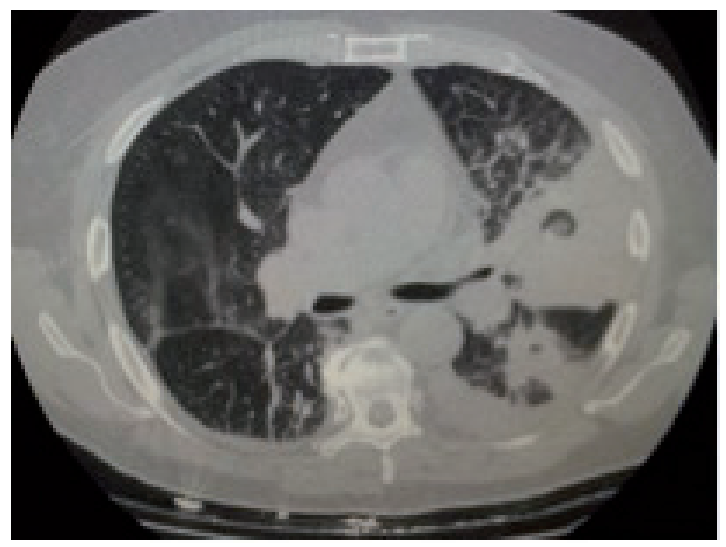

Figura 3. TC de control a los 10 días. Aumento de la lesión con cavitación central.

Paciente se fue de alta a su domicilio, el día $02 / 05 / 16$, en buenas condiciones generales, quedando con indicaciones de rehospitalización posteriormente para continuar con su quimioterapia de consolidación.

\section{DISCUSIÓN}

A excepción de cuando está disponible una muestra de tejido, el diagnóstico de API se basa en la asociación de factores del huésped, TC pulmonar característico, y los resultados microbiológicos que pueden ser detectados a través de cualquiera de las pruebas directas (tales como la microsco- pía directa o cultivo) o pruebas indirectas como el antígeno galactomanano (GM) o BD-glucano ${ }^{8}$. En los pacientes con neoplasias hematológicas, los biomarcadores se utilizan principalmente como pruebas de detección para inicio de tratamiento antifúngico precoz ${ }^{11}$.

Las recomendaciones del ECIL (European Conference on Infections in Leukaemia, 2012) para el tratamiento de las infecciones en pacientes hematológicos, están basadas en literatura acerca de pacientes en riesgo de enfermedad fúngica invasora $>5-10 \%$, es decir, pacientes con leucemia aguda con neutropenia prolongada. En relación al diagnóstico de API en estos pacientes, apoya firmemente el uso de GM (con nivel de evidencia A II). Para los ensayos de reacción en cadena de la polimerasa, la falta de estandarización entre los laboratorios de los procedimientos analíticos no permite recomendaciones para su uso clínico ${ }^{12}$.

El GM es un heteropolisacárido de la pared celular de Aspergillus sp y que durante la infección invasora tisular es el principal exoantígeno expresado en tejidos, sangre, orina, liquido cefalorraquideo y tracto respiratorio. Una de las pruebas ampliamente utilizadas para este propósito es Platelia $^{\text {TM }}$ Aspergillus EIA (GM-EIA), un inmunoensayo enzimático que detecta la presencia de este exoantigeno. El kit GM-EIA ha demostrado un buen rendimiento en el diagnóstico de aspergilosis invasora a partir de muestras de suero al utilizarse un índice de densidad óptica (IDO) de 0,5 como valor de corte. Se ha reportado una sensibilidad y especificidad aproximada de $78 \%$ y $81 \%$; respectivamente. Además de su sensibilidad, la prueba es muy reproducible cuando se comparan los resultados obtenidos entre distintos laboratorios ${ }^{13,14,15}$. Durante la infección, se describen 2 fases a nivel histológico, la angioinvasiva y la broncoalveolar. Cuanto más grave es la neutropenia del húesped, más rápido y probable es la invasión vascular, y más temprana y corta la fase broncoalveolar. 
Se ha visto correlación entre signos radiológicos que corresponden a esta fase y probabilidad de positividad en los test diagnósticos no invasivos, con mayor proporción de cultivos positivos de secreciones respiratorias en pacientes no neutropénicos (en los cuales la fase broncoalveolar es lo suficientemente larga), en neutropénicos, la fase de angioinvasión es más prolongada, por lo que es más probable que GM en suero sea positivo a diferencia de los no neutropénicos en que se retrasa la fase angioinvasora (o incluso no se produce en absoluto) y es menos probable que el GM sérico sea positivo ${ }^{11}$. Las imágenes radiológicas se han convertido en la piedra angular del diagnóstico de API, cuando los cambios pulmonares se desarrollan en pacientes neutropénicos con fiebre resistente a los antibióticos, sin embargo, sus hallazgos no son específicos. Algunos autores han identificado 2 patrones diferentes de API, basándose en la correlación entre la histología y patrones del TC: la fase angioinvasora se caracteriza por un nódulo con signo del halo y la fase broncoalveolar, que se caracteriza por destrucción de la pared bronquiolar por Aspergillus, micronódulos $(<1 \mathrm{~cm})$ centrolobulillares y opacidades en árbol en brote ${ }^{8}$. Infiltrados y macronódulos son cambios inespecíficos que representan consolidación temprana de la infección. La aparición de un nódulo pulmonar hemorrágico, denominado "signo del halo" es típico (pero no exclusivo) de API. Consiste en un centro nodular $\geq 50 \%$ (densa bola fúngica), rodeado de atenuación en vidrio esmerilado. El halo ocurre en la etapa inicial y está presente por un corto período (5 a 14 días) después de la aparición de API. Su espe- cificidad puede calcularse en $80 \%{ }^{16}$. El signo del aire creciente es un hallazgo de buen pronóstico debido a que marca la fase de recuperación de la infección. Tiene una sensibilidad de 48 a $68 \%$. Los nódulos están compuestos por tejido pulmonar hemorrágico infartado. A medida que los neutrófilos se recuperan y el paciente restablece su respuesta inmune, la reabsorción periférica de tejido necrótico causa la retracción del centro infartado y el aire llena el espacio comprendido entre ambas zonas. Esto crea una región de aire creciente dentro del nódulo y en la etapa tardía de API se puede formar una lesión cavitaria ${ }^{16,17}$.

La presencia de un nódulo con un signo del halo se ha demostrado que es muy sugerente de IPA en pacientes neutropénicos, sin embargo, hasta el $40 \%$ de los pacientes con API no se presentan con un nódulo con signo del halo ${ }^{8}$.

¿Cómo podemos hacer diagnóstico precoz de API? El primer paso es hacer monitoreo serológico seriado con GM 3 veces a la semana, y establecer criterios agresivos para solicitar TC de tórax. El segundo paso es reconocer las imágenes que preceden el signo del halo e interpretarlos como API temprana en el contexto de GM serológico positivo ${ }^{11,18}$.

En resumen, la aplicación de GM en suero seriado y una estrategia agresiva de TC de tórax temprana en pacientes neutropénicos pueden permitir a los médicos diagnosticar API precozmente, antes de la aparición del signo del halo. Los potenciales beneficios y costos de un enfoque de este tipo deben ser evaluados por estudios prospectivos ${ }^{11}$.

\section{REFERENCIAS}

1. Segal BH. Aspergillosis. $N$ Engl $J$ Med. 2009;360:1870-1884.

2. Latgé J P. The pathobiology of Aspergillus fumigatus. Trends Microbiol 2001; 9: 382-9.

3. Bulpa P, Dive A, Sibille Y. Invasive pulmonary aspergillosis in patients with chronic obstructive pulmonary disease. Eur Respir J 2007; 30: 782-800.

4. Gangneux JP, Camus C, Philippe B. Epidemiology of and risk factors for invasive aspergillosis in nonneutropenic patients. Rev Mal Respir 2008; 25: 139-53.

5. Fortún J, Meije Y, Fresco G. Aspergilosis. For- 
mas clínicas y tratamiento. Enferm Infecc Microbiol Clin. 2012;30(4):201-208.

6. Patterson TF, Kirkpatrick WR, White M, et al. Invasive aspergillosis: disease spectrum, treatment practices, and outcomes. I3 Aspergillus Study Group. Medicine 2000; 79:250-60.

7. Pagano L, Girmenia C, Mele L, et al. Infections caused by filamentous fungi in patients with hematological malignancies: a report of 391 cases by GIMEMA infection. Haematologica 2001; 86:862-70.

8. Bergeron A, Porcher R, Sulahian A. The strategy for the diagnosis of invasive pulmonary aspergillosis should depend on both the underlying condition and the leukocyte count of patients with hematologic malignancies. Blood. 2012;119(8):1831-1837.

9. Kousha M, Tadi R, Soubani O. Pulmonary aspergillosis: a clinical review. Eur Respir Rev 2011; 20: 121, 156-174.

10. Fortún J, Carratalá J, Gavaldác J., et al. Recomendaciones sobre el tratamiento de la enfermedad fúngica invasiva por Aspergillus spp. y otros hongos filamentosos de la Sociedad Espanola de Enfermedades Infecciosas y Microbiología Clínica. Enferm Infecc Microbiol Clin. 2011;29(6):435-454.

11. Nucci M, Nouér S, Cappone D., et al Early diagnosis of invasive pulmonary aspergillosis in hematologicpatients: an opportunity to improve the outcome. Haematologica 2013; 1657-1660. doi:10.3324/haematol.2013.094359.
12. Marchetti O, Lamoth F, Mikulska M. ECIL recommendations for the use of biological markers for the diagnosis of invasive fungal diseases in leukemic patients and hematopoietic SCT recipients. Bone Marrow Transplantation (2012) 47, 846-854.

13. Coria P. Galactomanano y aspergilosis invasora en niños. Rev. chil. infectol. 2008, vol.25, n.6, pp. 483-483. http://dx.doi.org/10.4067/S071610182008000600013.

14. Alvarez E. Utilidad de la prueba AspergillusLFD para el diagnóstico de aspergilosis: primera experiencia en Chile. Rev. chil. infectol. 2015, vol.32, n.1, pp. 117-119. http://dx.doi.org/10.4067/ S0716-10182015000200017.

15. Verweig PE, Erjavec Z, Sluiters W, et al. Detection of antigen in sera of patients with invasive aspergillosis: intra-and interlaboratory reproducibility. J Clin Microbiol 1998; 36: 1612-1616.

16. Reichenberger F, Habicht J.M., Gratwohl A., Tamm M. Diagnosis and treatment of invasive pulmonary aspergillosis in neutropenic patients. Eur Respir J 2002; 19: 743-755

17. Abramson S. The Air Crescent Sign. Radiology. 2001; 218:230-232. 39.

18. Sun K-S, Tsai C-F, Chen SC-C, Chen Y-Y, Huang W-C. Galactomannan Testing and the Incidence of Invasive Pulmonary Aspergillosis: A 10-Year Nationwide Population-Based Study in Taiwan. Chotirmall SH, ed. PLoS ONE.2016;11(2):e0149964.doi:10.1371/journal. pone. 0149964 . 